\title{
The Effect of Age on Oral Liquid Oxycodone Pharmacokinetics in Pediatric Patients.
}

\author{
Patcharee Sriswasdi, MD, MPH*, \#, Christine Dube, MS, BSN, RN ${ }^{*}$, Luis Pereira, PhD*, \\ Shannon Manzi, Pharm. D*, Charles Berde, MD*, Susan Goobie, MD, FRCPC*. \\ *Department of Anesthesiology, Critical Care, Perioperative and Pain Medicine, \\ Boston Children's Hospital and Harvard Medical School, Boston, MA. \\ \#Department of Anesthesiology, Siriraj Hospital, Mahidol university, Thailand.
}

Introduction: Oral liquid Oxycodone is one of the most common opioid used for pain control in children in United State. Studies have demonstrated marked variation in the pharmacokinetics (PK) of oxycodone among the pediatric population $(1,2)$ which may be due to genetic variation of its metabolism. Development also has significant effect on the expression of cytochrome P450 (CYP). Additionally, developmental changes in the activity of intestinal drug-metabolizing enzymes and transporters could potentially alter the bioavailability of oxycodone. Although $80 \%$ of oxycodone breakdown occurs by CYP3A4 into an inactive metabolite, up to $11 \%$ occurs through CYP2D6 into oxymorphone. Oxymorphone has 40 times the affinity and 8 times the potency of oxycodone upon Mu-opioid receptors. There are several studies that demonstrated the lower activity of CYP3A4 at birth and in neonates. This suggests the need to rationalize oxycodone dosing regimens in neonates, infants, and children. Understanding the effect of age on oral liquid oxycodone pharmacokinetics (PK) favors the safe and effective use of this analgesic in a wide variety of pediatric surgical patients. The aim of this study is to characterize the PK of oral liquid oxycodone and its metabolites (oxymorphone, noroxymorphone and noroxycodone) between 4 age groups: 0-6 months, 6-12 months,1-2 years and 2-6 years.

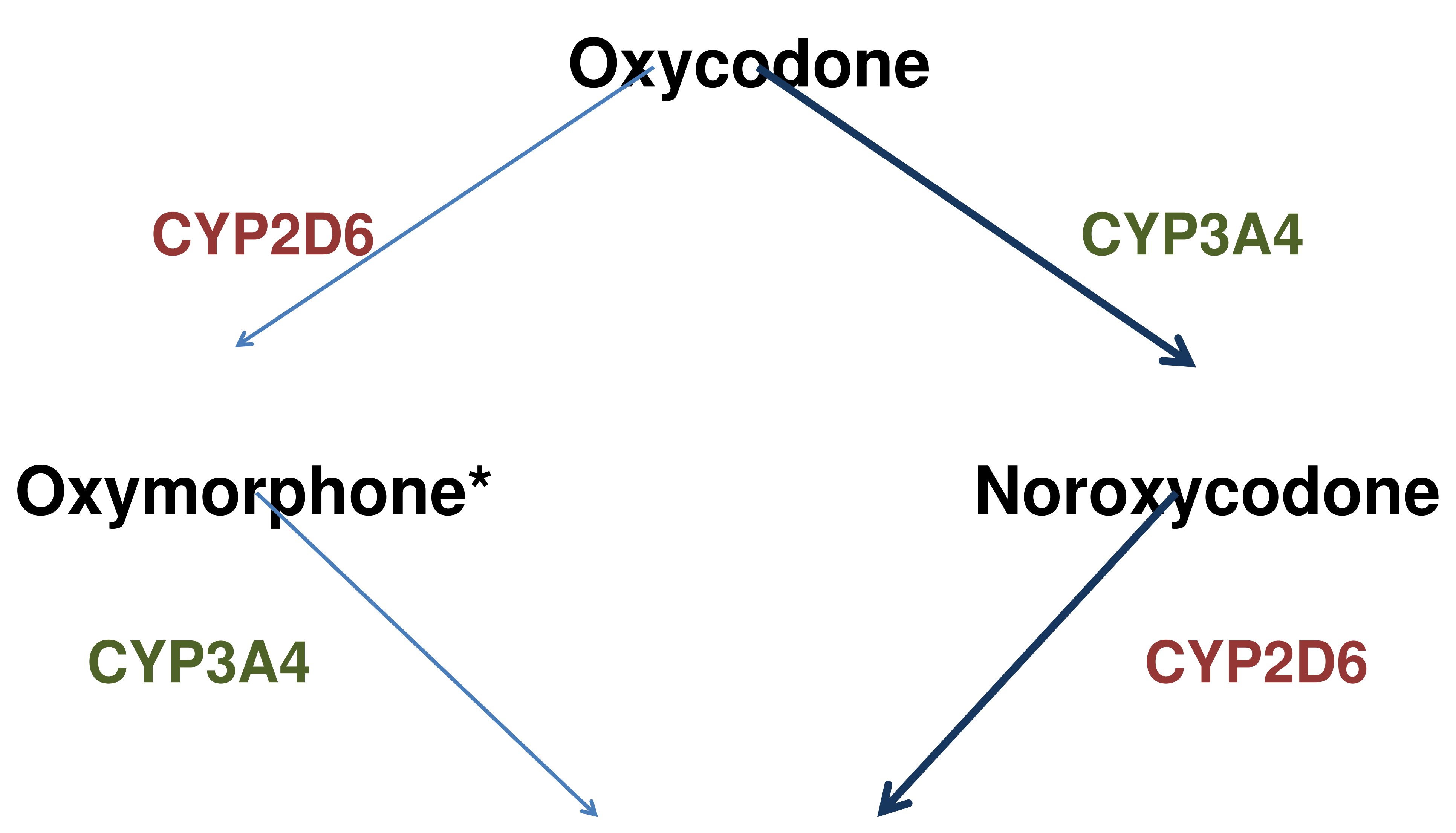

Noroxymorphone

Methods: This prospective cohort, single-center trial was approved by the hospital investigational review board. A total of 40 opioid-naive children, aged $0-6$ years $(n=10 ; 0-6$ months, $n=10 ; 6-12$ months; $n=10,1-2$ years; $n=10,2$ - 5 years), scheduled for in-patient surgery, have been consented. Blood samples were collected for the assay of oxycodone and its main metabolites. Oxycodone, oxymorphone, noroxymorphone and noroxycodone levels at 10 time points were assayed using liquid chromatography- mass spectrometry (UPLC/MS/MS) and single-dose pharmacokinetics parameters were determined.

Results: Concentration data revealed a substantial interpatient PK variability likely due to age differences (as shown in the graphs).

Discussion: A trend appears to exist with age contributing to the variability of $\mathrm{PK}$ of oxycodone. This justifies the need for its consideration in the dosing optimization of oxycodone based on individual age. It is possible that oxycodone metabolism might be different in the pediatric population when compared to adult.

Reference:1. Kokki et al. 2004 Clin Pharmacokinet, 43: 613-622.

2. El-Tahtawy et al. $2006 \mathrm{~J}$ Clin Pharmacol 46: 433.

Disclosure: The authors have no conflicts of interest. The work was supported internally by the Departments of Anesthesiology, Critical Care, Perioperative and Pain Medicine and the PKLab - Pharmacometrics Research Core, Boston Children's Hospital, Boston.

PKLab@childrens.harvard.edu
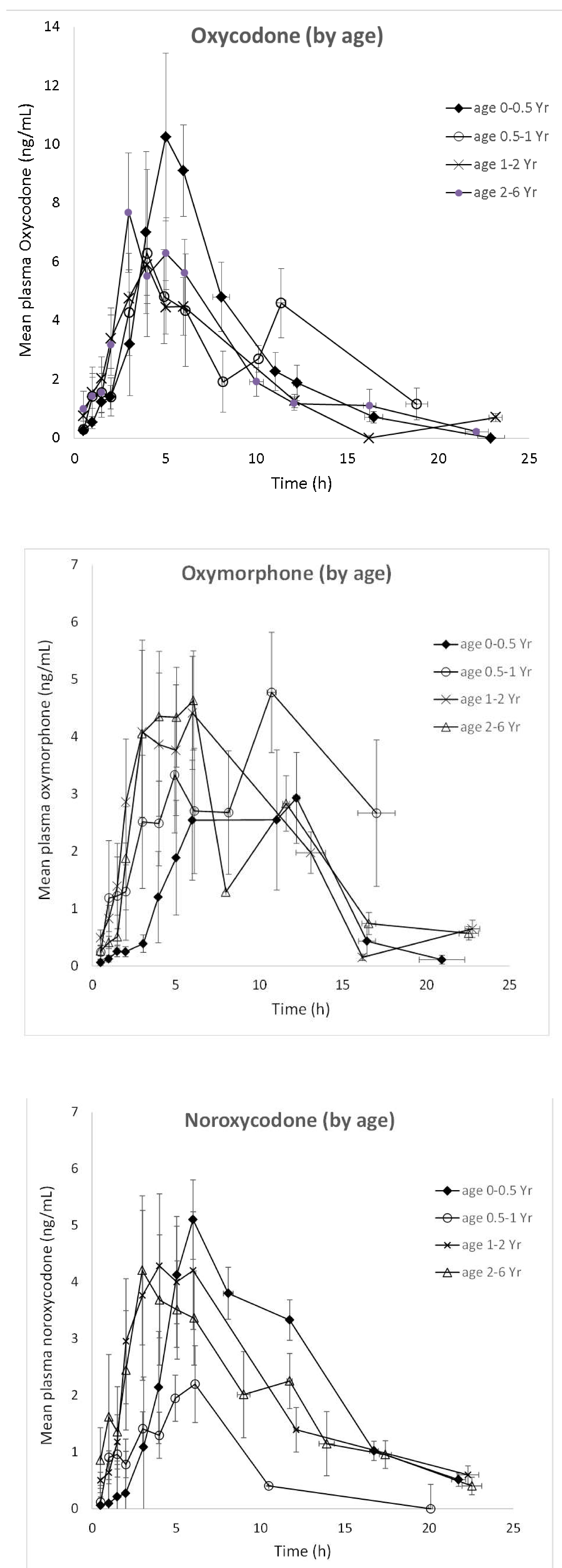

Boston Children's Hospital
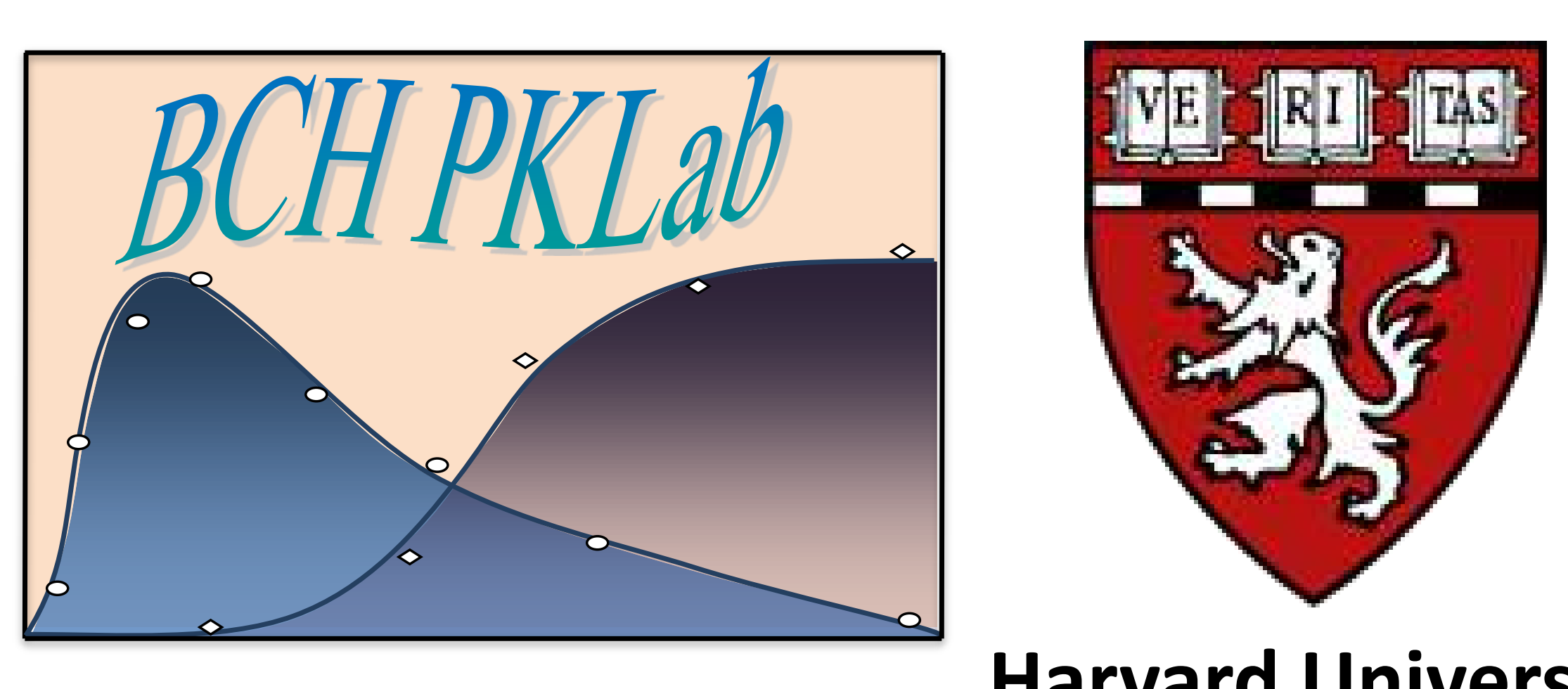

Harvard University 